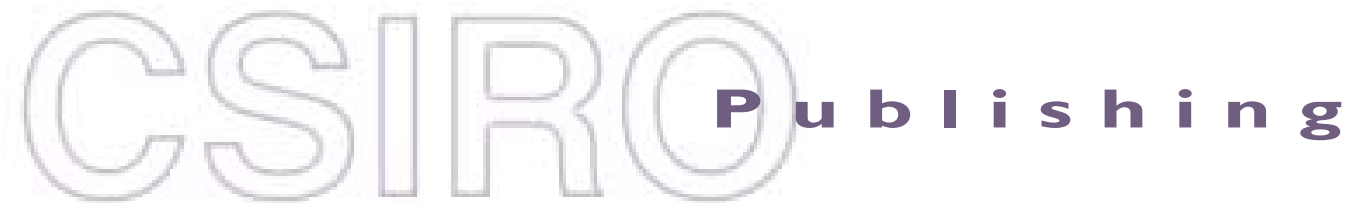

\section{Publications of the Astronomical Society of Australia}

Volume 19, 2002

(C) Astronomical Society of Australia 2002

An international journal of astronomy and astrophysics

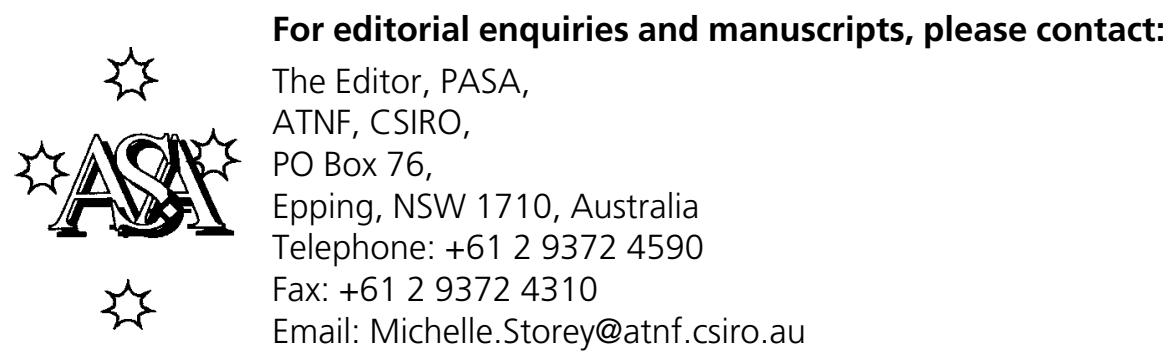

For general enquiries and subscriptions, please contact: CSIRO Publishing PO Box 1139 (150 Oxford St)

Collingwood, Vic. 3066, Australia

Telephone: +6139662 7666

Fax: +61 396627555

Email: publishing.pasa@csiro.au

C S I RO

PUBLISHING Published by CSIRO Publishing

for the Astronomical Society of Australia

www.publish.csiro.au/journals/pasa 


\title{
Models of Variability in Blazar Jets
}

\author{
Apostolos Mastichiadis ${ }^{1}$ and John G. Kirk $^{2}$ \\ ${ }^{1}$ Physics Department, University of Athens, GR-15784 Zografos, Athens, Greece \\ amastich@phys.uoa.gr \\ ${ }^{2}$ Max-Planck-Institut für Kernphysik, Postfach 1039 80, D-69029 Heidelberg, Germany \\ John.Kirk@mpi-hd.mpg.de \\ Received 2001 September 3, accepted 2002 March 13
}

\begin{abstract}
During the last decade multiwavelength observations of blazars have revealed many interesting patterns in their emission across the EM spectrum. In the present article we will review the time-dependent one-zone models and the models which advocate an acceleration and a radiation zone, and we will make some comparisons between them, especially in light of recent observations of the so-called $\mathrm{TeV}$ blazars.
\end{abstract}

Keywords: acceleration of particles — radiation mechanisms: non-thermal — shock waves — galaxies: active

\section{Introduction}

During the last decade there has been a drastic change in our picture of high energy emission from active galactic nuclei (AGN). The 3rd CGRO catalogue lists over 60 AGN as high confidence sources of $\mathrm{GeV}$ gamma rays (Hartman et al. 1999) while at least two AGN, Mkn 421 and Mkn 501, have been detected in the TeV regime (Punch et al. 1992; Quinn et al. 1996), see also Horan et al. (2002) for a recent possible discovery of $1 \mathrm{H} 1426+428$. All of these AGN belong to the category of blazars, which include OVV and flat radio sources, many of which exhibit superluminal motion.

On the theoretical front it became quickly apparent that the gamma-ray emission was connected with processes in the jet rather than in the core. While this general picture remains more or less undisputed, many models have been proposed for the high energy emission itself; these can be roughly divided into leptonic or hadronic in origin, depending on whether it is electrons or protons which are responsible for the gamma-ray emission. Thus while there are models which invoke protons as the ultimate source of high energy emission (Mannheim 1993; Protheroe 1997), the majority of the proposed models assume that the gamma rays come from inverse Compton scattering of relativistic electrons on some soft photon targets. The source of these targets is still an open question and many possible origins have been proposed, such as accretion disk photons (Dermer, Schlickeiser, \& Mastichiadis 1992), diffuse isotropic photons coming from regions such as the broad line clouds (Sikora, Begelman, \& Rees 1994), internally produced synchrotron photons (Maraschi, Ghisellini, \& Celotti et al. 1992; Marscher \& Travis 1996; Inoue \& Takahara 1996), or combinations thereof (Dermer, Sturner, \& Schlickeiser 1997), with each model giving rather similar spectral features and characteristics.

A very interesting aspect which emerged from the intense gamma-ray monitoring of the sources was the discovery of fast variability. So in addition to the already known variability of many sources in the lower energy bands (for a review see Wagner \& Witzel 1995), the blazar Mkn 421 was discovered to exhibit TeV flares, the fastest of which had a duration of about 15 minutes (Gaidos et al. 1996), while in the case of Mkn 501 the reported variability is about one day (Aharonian et al. 1999). More powerful sources, such as $3 \mathrm{C} 279$, have shown variability in the GeV regime of the order of an hour (Hartman et al. 1996). These observations put new, interesting constraints on the theoretical models of high energy emission from AGN since one expects the particle cooling times to be of the order of the flare duration itself. The imposed constraints become even tighter from recent results of multiwavelength campaigns which show certain trends in the evolution of flares along the EM spectrum. Thus Mkn 421 was discovered to exhibit quasisimultaneous variation in the $\mathrm{keV}$ and $\mathrm{TeV}$ regime (Macomb et al. 1995), while other energy regimes (most notably the $\mathrm{GeV}$ regime) remained virtually unaffected. The other AGN detected in TeV, Mkn 501, has shown similar trends (Catanese et al. 1997; Pian et al. 1998; Catanese \& Sambruna 2000).

The aforementioned observations provoked a flurry of models which addressed explicitly either the fast variability (Salvati, Spada, \& Pacini 1998), the multiwavelength spectrum (Ghisellini, Maraschi, \& Dondi 1997), or both (Mastichiadis \& Kirk 1997). In Section 2 we will review the basic features of such models especially in the context of the so-called homogeneous synchrotron self-Compton models (SSC). In Section 3 we will address explicitly the problem of particle acceleration and present a simple way one can explain certain observations with the picture of accelerating/radiating particles.

\section{Homogeneous Synchrotron Self-Compton Models}

This class of models, based on the ideas first put forward by Jones, O'Dell, \& Stein (1974), assumes that the radiation is produced in a homogeneous spherical 
region containing magnetic fields and relativistic electrons (Inoue \& Takahara 1996; Ghisellini et al. 1996; Mastichiadis \& Kirk 1997, henceforth MK97). The flaring activity of the sources implies that, in order to gain insight into the conditions of the source, time-dependent calculations are required rather than a set of spectra at steady state. In order to address explicitly the temporal behaviour of the spectrum, MK97 used a set of time-dependent, spatially averaged kinetic equations for the electrons and photons adopting the approach outlined in Mastichiadis \& Kirk (1995). The electrons are assumed to have a power-law uniform injection in a spherical source (blob) of radius $R$; the blob itself is supposed to move at some small angle $\theta$ to our line of sight with a bulk Lorentz factor $\Gamma$. The electrons lose energy from synchrotron radiation on a magnetic field of strength $B$ and from inverse Compton radiation on the synchrotron photons produced. The electron distribution function so obtained is then convolved with the single electron synchrotron and inverse Compton emissivities, and the overall photon spectrum is obtained after allowing for the possibility of photon-photon pair production - a process which turns out to be negligible for the parameters used.

Seven independent parameters are needed to determine a stationary spectrum in this model. They are the Doppler boosting factor $\delta=[\Gamma(1-\beta \cos \theta)]^{-1}$ (with $\beta c$ the bulk velocity of the source), the size of the source $R$, its magnetic field $B$, the mean time during which particles are confined in the source $t_{\mathrm{esc}}$, and three parameters determining the injected relativistic electron distribution: its luminosity or compactness $\ell_{\mathrm{e}}$, the spectral index $s$, and the maximum Lorentz factor of the electron distribution $\gamma_{\max }$. The inclusion of the particle escape time $t_{\mathrm{esc}}$ becomes necessary from the fact that the photon spectrum is rather flat between the radio and the infrared region, implying that the radiating particles do not have time to cool significantly.

Figure 1 shows a fit to the multiwavelength spectrum of Mkn 421 as given in Macomb $(1995,1996)$. The fit to the low state was obtained for $R=4.7 \times 10^{16} \delta_{15}^{-3} \mathrm{~cm}$, $B=0.07 \delta_{15} \mathrm{G}, \gamma_{\max }=2 \times 10^{5} \delta_{15}^{-1}, s=1.7, \ell_{\mathrm{e}}=1.9 \times$ $10^{-5} \delta_{15}^{-1}$, and $t_{\mathrm{esc}}=50 t_{\mathrm{cr}} \delta_{15}^{2}$. This leaves only one free parameter which can be suitably chosen as either the Doppler factor of the blob $\delta$ or the timescale over which variability can be observed $t_{\mathrm{var}}$ (in seconds). These two quantities are related, in this particular case, by the scaling relation $\delta=267 t_{\mathrm{var}}^{-1 / 4}$ ( $t_{\mathrm{var}}$ expressed in seconds). For the reported variability of about one day $\left(t_{\mathrm{var}}=10^{5} \mathrm{sec}\right)$ one readily finds $\delta=15$, which is close to the usually assumed values of the Doppler factor. As can be seen from Figure 1, the multiwavelength spectrum of the Mkn 421 flare can be fitted in a time-dependent fashion (i.e. before complete cooling can be achieved) by changing $\gamma_{\max }$ by a factor of about 5 . These changes result in large variations in the $\mathrm{X}$ and $\mathrm{TeV}$ regime (i.e. the cutoffs of the synchrotron and inverse Compton spectra) but are not especially prominent at other frequencies. An alternative

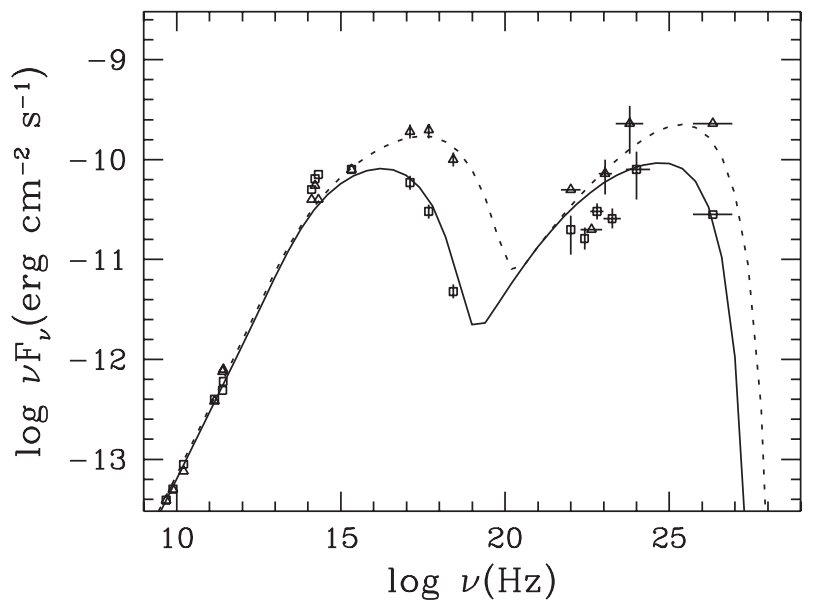

Figure 1 Low and high states of the multiwavelength spectrum of Mkn 421 obtained with the one-zone synchro-Compton models. The data points were taken from Macomb et al. (1996). For the parameters used see text.

way of producing a flare is to consider an increase in the luminosity of the injected relativistic electrons while leaving the other parameters unchanged. However the flares so produced do not seem to fit the particular observations (see MK97).

A rather tight constraint is placed on these models by recent X-ray observations of Mkn 421 (Krawczynski et al. 2001) which show the variability to be of the order of hours rather than days - the same timescale for variability was also seen in TeV $\gamma$-rays (Gaidos et al. 1996). To fit with the one-zone models, for these variations one needs to invoke Doppler factors of order 50 (Krawczynki et al. 2001 - the same result is derived from the MK97 calculations if one is to replace the day-long variability timescale used there with a sub-hour one $\left(t_{\mathrm{var}} \simeq 10^{3} \mathrm{sec}\right)$; then the relation $\delta=267 t_{\mathrm{var}}^{-1 / 4}$ which was quoted earlier gives $\delta \simeq 50$ ). This is a rather high Doppler factor value, certainly much higher than those indicated by observations of apparent superluminal motion (Vermeulen \& Cohen 1994).

A second, more severe, problem that the one-zone models face is the counter-clockwise evolution of the intensity versus spectral index plot observed occasionally from some sources during their flaring state (for a recent review of the observations see Takahashi 2000). As the one-zone models do not treat acceleration of electrons but rather inject them to high energies, any flaring activity is produced from the cooling of the electrons and thus it always follows a clockwise pattern. Therefore, if the observations are confirmed, they will most probably rule out these models. We will come to this point in more detail in the next section.

\section{Particle Acceleration in Blazar Jets}

The one-zone models cannot treat acceleration as most theories predict an acceleration timescale $t_{\mathrm{acc}} \ll t_{\mathrm{cr}}=$ $R / c$. In a series of papers (Kirk, Rieger, \& Mastichiadis 1998, henceforth KRM; Kirk \& Mastichiadis 1999; 
Mastichiadis, Georganopoulos, \& Kirk 2000) have examined the effects of shock acceleration in blazar jets. For this they considered a thin shockwave moving down a cylindrically symmetric jet (Marscher \& Gear 1985) with a velocity $u_{\mathrm{s}}$ in the rest frame of the jet. The particles are accelerated by the shock through a first order Fermi scheme and subsequently escape downstream where they radiate (Ball \& Kirk 1992; Drury et al. 1999). Therefore the electron distribution function is given by the solution of two coupled differential equations for the acceleration and downstream region (cf. KRM equations 1 and 6). Thus the concept of the 'acceleration zone' as presented here, differs from the emission region in the homogeneous model discussed in the previous section, in that particles are injected at low energy and continuously accelerated to high energies. A further difference comes from the fact that the high energy cutoff of the electron distribution is given now by a detailed balance between the acceleration and loss rates at the Lorentz factor $\gamma_{\max }=1 /\left(\beta_{\mathrm{s}} t_{\mathrm{acc}}\right)$, where $\beta_{\mathrm{S}}$ is the corresponding synchrotron and inverse Compton rate. For $\gamma<\gamma_{\max }$ the acceleration rate exceeds the synchrotron loss rate while for $\gamma>\gamma_{\max }$ the distribution vanishes.

Once again the synchrotron and inverse Compton spectra are obtained by convolving the electron distribution function with the corresponding emissivities. However in this case one needs to integrate the differential electron density over the spatial coordinate since, in contrast to the homogeneous models, the acceleration region is distinct from the cooling region.

\subsection{Spectral Signatures of Acceleration}

As in the case of the homogeneous models one has first to seek parameters that could fit specific blazar spectra in a quiescent state and then induce a flare by changing some parameter of the fit. Perhaps the simplest way for that is to consider an increase in the number of particles injected in the acceleration mechanism. This can be considered as the case when the shock front overruns a region in the jet in which the local plasma density is enhanced. An increase of the injection rate by a factor $1+\eta_{\mathrm{f}}$ for a time $t_{\mathrm{f}}$ is found by setting

$$
\begin{array}{ll}
Q(t)=Q_{0} & \text { for } t<0 \text { and } t>t_{\mathrm{f}} \\
Q(t)=\left(1+\eta_{\mathrm{f}}\right) Q_{0} & \text { for } 0<t<t_{\mathrm{f}} .
\end{array}
$$

Figure 2 shows the evolution of the intensity and spectral index of a flare as the one described above when the observational window is away from the upper cutoff. For example, if the cutoff is chosen to be in the $100 \mathrm{keV}$ region, as in the case of Mkn 501 (Pian et al. 1998), then the spectral index plotted is calculated from the fluxes at 1 and $5 \mathrm{keV}$. When plotted against the flux at the lower frequency, the spectral index exhibits a characteristic looplike pattern, which is tracked in the clockwise sense by the system. This type of behaviour is well known and has been observed at different wavelengths in several sources e.g. OJ287, PKS 2155-304, and Mkn 421 (for a review see Takahashi 2000). It arises whenever the slope is controlled by synchrotron cooling so that information about injection propagates from high to low energy (Tashiro et al. 1995).

$$
\begin{aligned}
& \text { Duration }=10 \\
& \eta_{\mathrm{f}}=1 \\
& \nu_{1} / \nu_{\max }=0.01 \\
& \nu_{2} / \nu_{\max }=0.05
\end{aligned}
$$
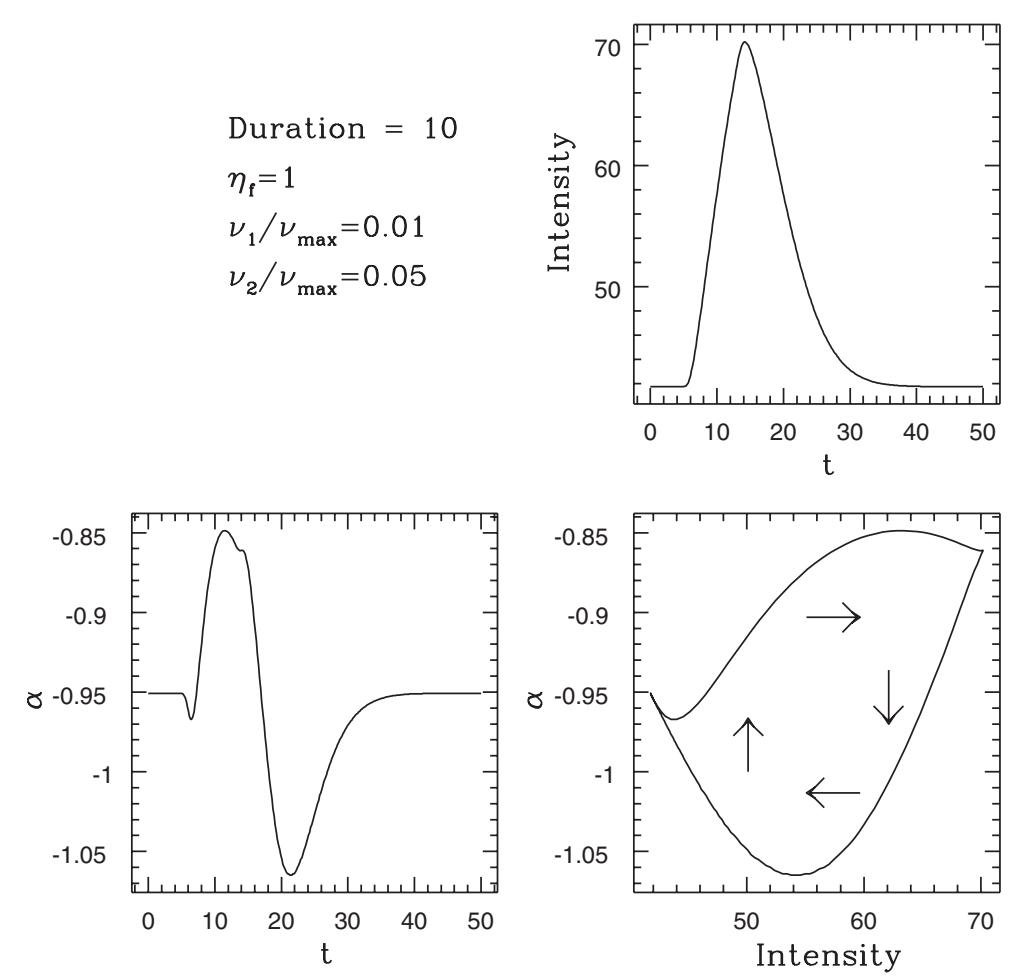

Figure 2 The intensity and spectral index during the flare described by equations (1) and (2) as a function of time at frequencies away from the high energy cutoff. Here $\eta_{\mathrm{f}}=1, t_{\mathrm{f}}=10 t_{\mathrm{acc}}$, and $u_{\mathrm{s}}=c / 10$. The loop in the $\alpha$ vs. intensity plot is followed in the clockwise direction. 


$$
\begin{aligned}
& \nu_{1} / \nu_{\text {max }}=0.18 \\
& \nu_{2} / \nu_{\text {max }}=0.9
\end{aligned}
$$
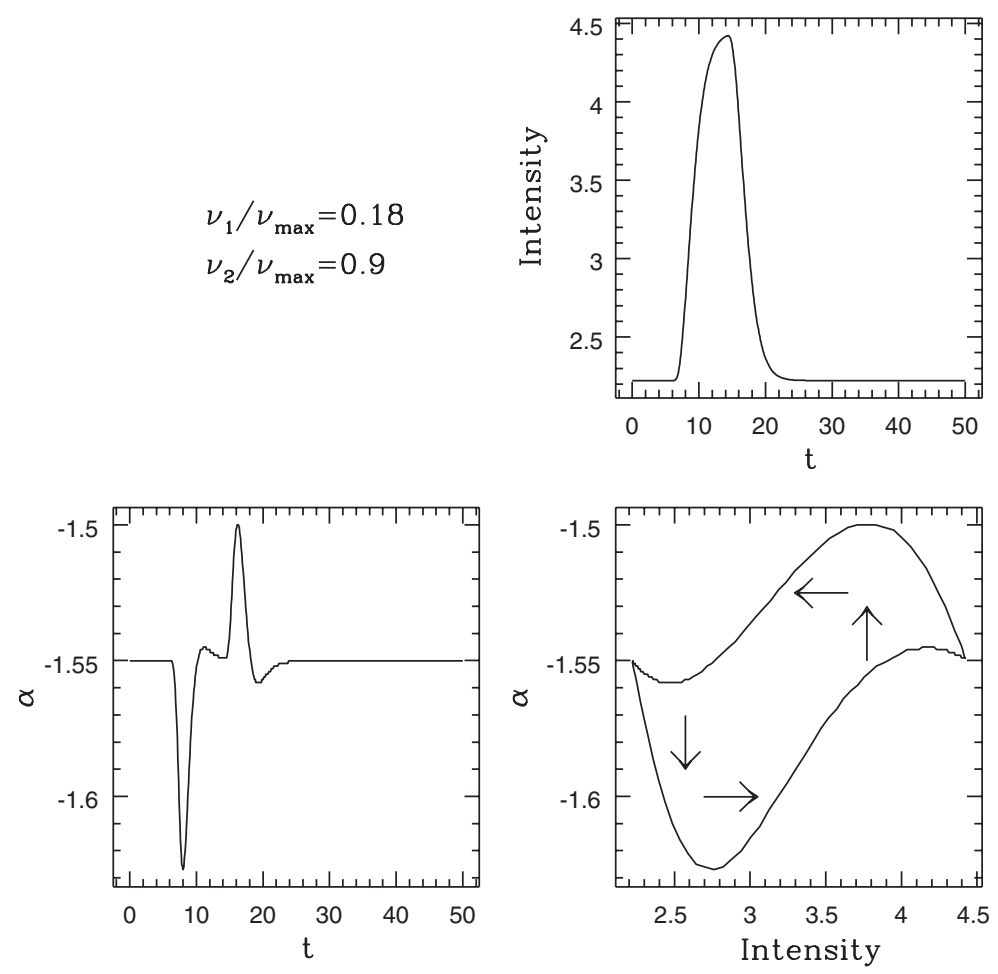

Figure 3 The evolution of the same flare as in Figure 2 but observed at frequencies close to the high energy cutoff. The loop in the $\alpha$ vs. intensity plot is followed in the anticlockwise direction.

If the system is observed closer to the maximum frequency, where the cooling and acceleration times are equal, the picture changes. Here information about the occurrence of a flare propagates from lower to higher energy, as particles are gradually accelerated into the radiating window. Such behaviour is depicted in Figure 3, where the same flare is shown at energies which are an order of magnitude higher than in Figure 2 (corresponding to 18 and $90 \mathrm{keV}$ in the case of Mkn 501). This time the loop is traced anticlockwise.

The flare behaviour in the $\mathrm{TeV}$ regime is similar to the one in the X-ray regime. This is because a single population of electrons produces the radiation in both bands. The only difference between them is that Klein-Nishina effects make the spectrum in the $\mathrm{TeV}$ regime somewhat steeper — for more details see Mastichiadis et al. 2001.

\section{Summary}

In this paper we have presented a selective account of recent results on AGN variability within the context of (a) homogeneous SSC and (b) diffusive particle acceleration models. We have shown that the SSC models give good overall fits to the multiwavelength $\mathrm{TeV}$ blazar spectra and can explain the major flares of these objects such as the ones reported by Macomb et al. (1995) and Pian et al. (1998), respectively, by increasing only one parameter of the fit, namely the high energy cutoff of the injected electron distribution. This type of flare is especially prominent at the high end of the photon distribution, i.e. in the $\mathrm{X}$-ray and TeV regime, leaving other energy regimes (most notably $\mathrm{GeV}$ gamma rays) practically unaffected, giving thus an explanation of why EGRET detected neither of these two major outbursts.

The very fast variation of the source Mkn 421, however, as reported by Gaidos et al. (1996) and Krawzynski et al. (2001) poses constraints for the homogeneous SSC models: in order for the models to satisfy simultaneously i) the high total luminosity, ii) the very fast variability, and iii) the transparency to $\mathrm{TeV}$ radiation (Bednarek \& Protheroe 1997), one needs either to invoke a high value of the Doppler boosting factor or to abandon the assumptions about a spherical source in favour of a laminar source geometry.

This picture can lead naturally to the shock-in-jet model, i.e. to the picture of a shock advancing down a jet, accelerating particles at the same time. This approach improves upon the assumptions of the homogeneous SSC model, as presented in Section 2, mainly by replacing the instantaneous electron injection with the concept of an acceleration timescale. It is therefore the interplay between the acceleration and energy loss timescales that provides us with the different flare behaviour shown in Figures 2 and 3 (for more examples of this the reader is referred to Kirk et al. 1999).

\section{Acknowledgments}

AM would like to thank the organisers of the workshop for their hospitality. This work was supported by the European Commission under the TMR program, contract number FMRX-CT98-0168. 


\section{References}

Aharonian, F. A., et al. 1999, A\&A, 349, 29

Ball, L. T., \& Kirk, J. G. 1992, ApJ, 396, L39

Bednarek, W., \& Protheroe, R. J. 1997, MNRAS, 292, 646

Catanese, M., \& Sambruna R. 2000, ApJ, 534, L39

Catanese, M., et al. 1997, ApJ, 487, L143

Dermer, C. D., Schlickeiser, R., \& Mastichiadis, A. 1992, A\&A 256, L27

Dermer, C. D., Sturner, S. J., \& Schlickeiser, R. 1997, ApJS, 109, 103

Drury, L. O'C., et al. 1999, A\&A, 347, 370

Gaidos, J. A., et al. 1996, Nature, 383, 318

Ghisellini, G., Maraschi, L., \& Dondi, L. 1996, A\&AS, 120, 503

Hartman, R. C., et al. 1996, ApJ, 461, 698

Hartman, R. C., et al. 1999, ApJS, 123, 203

Horan, D., et al. 2002, ApJ, in press

Inoue, S., \& Takahara, F. 1996, ApJ, 463, 555

Jones, T. W., O’Dell, S. L., \& Stein, W. A. 1974, ApJ, 188, 353

Kirk, J. G., \& Mastichiadis, A. 1999, APh, 11, 45

Kirk, J. G., Rieger, F. M., \& Mastichiadis, A. 1998, A\&A, 333, 452 (KRM)

Krawczynski, H., et al. 2001, ApJ, 559, 187

Macomb, D. J., et al. 1995, ApJ, 449, L99

Macomb, D. J., et al. 1996, ApJ, 459, L111 (Erratum)
Mannheim, K. 1993, A\&A, 269, 67

Maraschi, L., Ghisellini, G., \& Celotti, A. 1992, ApJ, 397, L5

Marscher, A. P., \& Gear, W. K. 1985, ApJ, 298, 114

Marscher, A. P., \& Travis, J. P. 1996, A\&AS, 120, 537

Mastichiadis, A., Georganopoulos, M., \& Kirk, J. G. 2001, in High Energy Gamma-Ray Astronomy, AIP Conf. Proc. 558, ed.

F. A. Aharonian \& H. J. Völk (New York: American Institure of Physics), 688

Mastichiadis, A., \& Kirk, J. G. 1995, A\&A, 295, 613

Mastichiadis, A., \& Kirk, J. G., 1997, A\&A, 320, 19 (MK97)

Pian, E., et al. 1998, ApJ, 492, L17

Protheroe, R. J. 1997, ASP Conf. Ser. 121, 585

Punch, M., et al. 1992, Nature, 358, 477

Quinn, J., et al. 1996, ApJ, 456, 83

Salvati, M., Spada, M., \& Pacini, F. 1998, ApJ, 495, L19

Sikora, M., Begelman, M. C., \& Rees, M. J. 1994, ApJ, 421, 153

Takahashi, T. 2000, in High Energy Gamma-Ray Astronomy, AIP Conf. Proc. 558, ed. F. A. Aharonian \& H. J. Völk (New York: American Institute of Physics), 346

Tashiro, M., et al. 1995, PASJ, 47, 131

Vermeulen, R. C., \& Cohen, M. H. 1994, ApJ, 430, 467

Wagner, S. J., \& Witzel, A. 1995, ARA\&A, 33, 163 